\title{
Medical Informatization based on Cloud Computing
}

\author{
Xiuxia $\mathrm{Yu}^{1}$ and Jing $\mathrm{Bian}^{2}$ \\ ${ }^{1,2}$ College of Computer Science and Technology Changchun University \\ Changchun, China, 130022 \\ 434218708@qq.com ${ }^{1}, 583745233 @ q q . c^{2}{ }^{2}$
}

\begin{abstract}
Technology and concept of cloud computing have changed the traditional application pattern of computing resources. Here we briefly introduce the concept and typical applications of cloud computing, and put forward the software services based on cloud computing technology in medical informatization and the application of a cloud storage service model. We also discuss the challenges faced in the application of the cloud computing technology during medical informatization.
\end{abstract}

Keywords: Cloud computing; Medical informatization; Software services; Cloud storage service

\section{Introduction}

Medical service informatization is a global trend. With the rapid development of information technology, a growing number of hospitals in China are speeding up the overall construction of information-based platforms and Hospital Information System (HIS), aiming to improve the service level and core competitiveness. Informatization improves not only the work efficiency of doctors, but also the satisfaction and trust of patients. Thus, the gradual integration between medical business applications and basic network platform becomes a new trend for the informatization of hospitals in China, especially large- and medium-sized hospitals. Medical informatization is defined as the digitization, networking and informatization of medical services. Sepcifically, computer science, modern network communication technology and database technology are applied into the collection, storage, processing, extraction and exchange of patient information and management information between hospitals and between departments of a hospital, thus satisfying the functional requirements of all authorized users. The cloud computing technology brings a powerful, flexible and low-cost cooperation \& innovation platform for medical informatization.

Specifically, all computing resources are integrated and automatically managed on software, without human participation. Equivalently, the computing power can be used as a circulating commodity in a convenient and cheap way like coal gas, water and electricity. Thus, medical and health enterprises can build informatization through the purchase of computing power and without construction of relevant facilities. In this way, hospitals do not have to worry about the tedium, but focus on the improvement of doctor business level, which is favorable for innovation. The appearance of cloud computing brings bran-new knowledge about medical informatization.

\section{Cloud Computing}

\subsection{Definition of Cloud Computing}

National Institute of Standards and Technology (NIST) defines cloud computing as a universal, convenient, and on-demand mode for online access to configurable shared 
computing resources (e.g. networks, servers, storage, applications and services). The computing resources can be rapidly provided and released with the least amount of management expense and the lowest intervention of service providers [1]. In other words, cloud computing, with the virtualization technology as the basis and networks as the carrier, is a super computing mode of collaborative works that provides infrastructure platform, software services and integrates large-scale extendible dispersed computing resources (e.g. computation, storage, data, applications). As a brand-new mode of Internet applications, cloud computing will become the dominant way of service and information acquisition.

Fundamentally, cloud computing is a novel commercial mode of data center operation. Thus, cloud computing has two basic contents:

(1) It describes the infrastructure for construction of application programs;

(2)It describes the application and expanded services that are built upon such infrastructure.

The final objectives of cloud computing are to provide computation, services and applications as public facilities, so that the public can use these computer resources like water, electricity, coal gas and telephone.

\subsection{Characteristics of Cloud Computing [1]}

(1) Virtualization. The virtualization technology separates application software and special hardware, and thus offer cloud service suppliers with the control ability for management of abundant server-based workload.

(2) Geo-distribution. The data center under multiple geo-distributions allows the cloud suppliers to flexibly allocate the load unto the resource that is nearest to the terminal user.

(3) Elasticity computing. Hardware devices, such as hard disks, would finally become exhausted and useless, owing to understandable physical reasons. Along with the growth of hardware resource pool, the possibility of any hardware facility becoming useless within any week, day or hour is increasing. Thus, the cloud computing applications and infrastructure should be designed to be capable of providing routine detection, analysis and recovery services.

(4) Advanced safety. Computing cloud is a major target for virtual space criminals and others who aim to cause chaos. Thus, advanced safety technology, tools and strategies are necessary safeguard that ensures that malicious individuals or organizations cannot invade clouds or damage application services or data.

(5) Large-scale. A successful cloud deployment that reduces costs by maximizing the operational efficiency must be large-scale.

(6) Identity. To maximize the operational efficiency, a successful cloud deployment must restrict the scopes of the hardware, infrastructure, software platform, strategy and flow that it supports.

\subsection{Service Mode of Cloud Computing [2]}

Cloud computing mainly has three service modes: Infrastructure as a Service (IaaS), Platform as a Service (PaaS), and Software as a Service (SaaS). Logically, the cloud computing service modes are located above the IP network infrastructure, which connects the customer and the applications at the cloud service end.

(1) IaaS: It provides consumers with powers by offering process, storage, network and other basic computing resources, so that consumers can deploy and run any software. The consumers do not manage or control the bottom cloud infrastructure, but control the applications of operational system and storage deployment, and can also regulate the selected network parts (e.g. mainframe fireproofing walls) finitely. IssS includes computation, storage, content distribution into network, backup, and recovery. The 
advantages of IaaS are that consumers only need low-cost hardware, and rent the corresponding computing power and storage power as needed, which largely reduces the hardware-related costs.

(2) PaaS: It provides the following powers to consumers: consumers deployed in the cloud infrastructure can create applications independently, or via the program language or tools provided by cloud suppliers. Cloud customers do not have to manage or control the network, server, operational system or storage at the bottom of cloud infrastructure, but could regulate the application of deployment and the allocation of potential application operational environment. PaaS provides enterprises or individuals with the intermediate platform for research and development, and offers the development of application programs, database, application server, tests, trusteeship, and application services.

(3) SaaS: It provides consumers with power by using the applications on the cloud infrastructure offered by the suppliers. Customers do not have to manage or control the network server, operational system or storage to the bottom of cloud infrastructure. SaaS includes the E-mail and office products, customer relationship management (CRM), enterprise resource planning (ERP), social networks, collaborative works, and management of documents and contents. The advantages of this service mode are: the service supplier maintains and manages software, offers the hardware facilities for software running, while the consumer can use the software at anytime and anywhere as long as he/she is endowed with access to the Internet terminal. This is the most costeffective operational mode for network applications. For small-sized enterprises, SaaS is the optimal pathway to acquire advanced technology.

\subsection{System Framework of Cloud Computing Platform}

Cloud computing platform can be a variety of resources together, "a dynamic allocation of computer system resources pool", software, hardware, data, information services, etc. can be used in the cloud computing platform for leasing. Fig. 1 is the commonly used "5-4-3" concept model for cloud computing platform. Cloud computing platform has Flexible service, On-demand service, Ubiquitous access, Service can be charged, Resource pool etc. 5 characteristics, public cloud, private cloud, hybrid cloud, community cloud etc. 4 deployment models, infrastructure as a service, software as a service, platform as a service etc, 3 service model. 


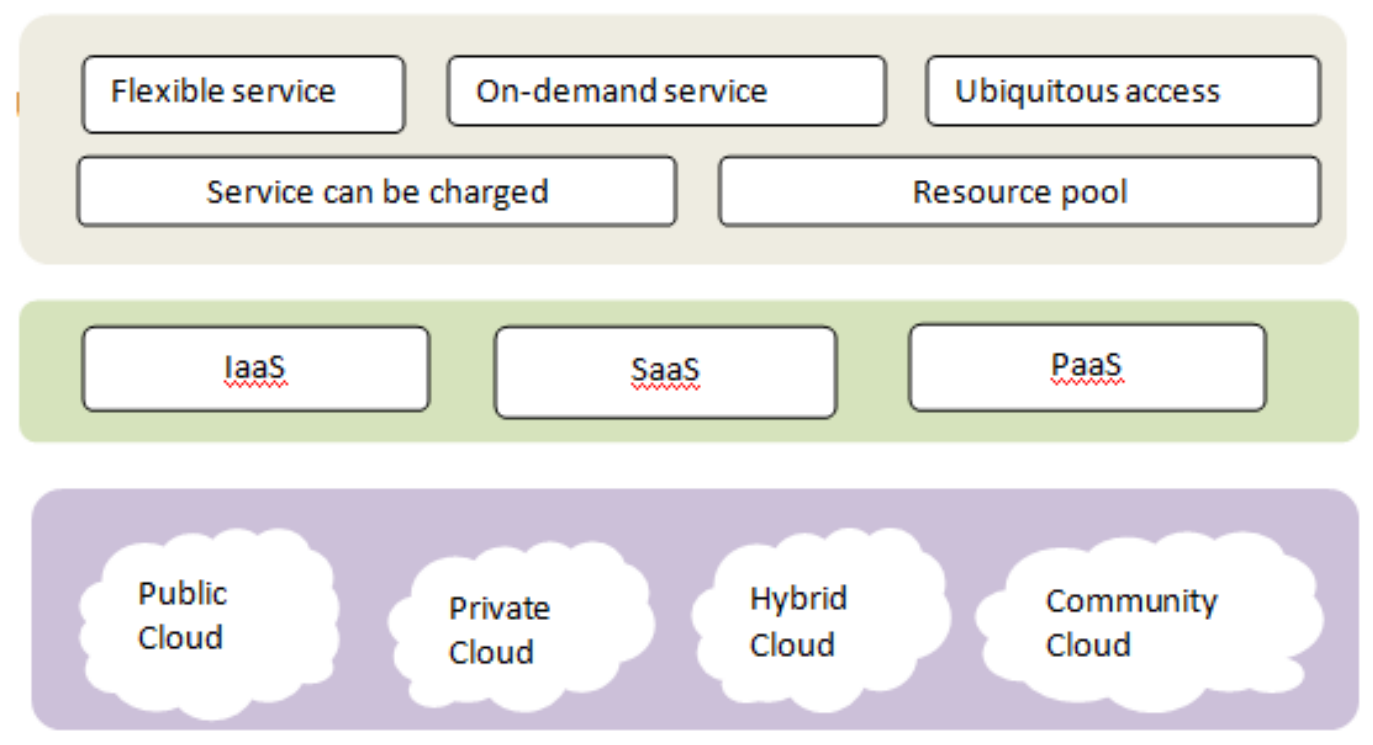

Figure 1. Cloud Computing Platform "5-4-3" model

\section{Application of Cloud Computing into Medical Informatization}

\subsection{Cloud Computing to Achieve the Medical Information in the Field of the Five" Unified"[3]}

(1) Unified data management center. Through data centralized storage, virtualization management and other technical means, the construction of a unified medical cloud data center.

(2) Unified service development platform. Cloud computing platform services technology, including data support, general technology support and industry applications support, including a unified service delivery. And the establishment of hospitals, community and other types of health services and medical and health resources integration, for the health service to provide integration support, set the standard, improve the quality of medical data, promote health information sharing and collaborative diagnosis, medical resource sharing.

(3) Unified application and deployment center. In the traditional medical and health information based, public health, regional health, integrated health management, health services, and other several health services in the field of information system step by step to full migration to the medical cloud platform, the establishment of service to regional health, public health and health tube of cloud services, applications, application deployment, delivery of cloud model application.

(4) Unified resource scheduling center. Through the virtual pool and distributed computing technology, it resources of server, CPU, storage devices, and IO devices and network bandwidth for unified management, and then greatly enhance the efficiency in the use of IT resources, form a unified and efficient resource scheduling center.

(5) unified security monitoring center. The cloud security technology, make full use of the cloud the powerful computation ability achieve cloud model of security detection and protection, formed unified security monitoring and service center with cloud model without boundary security door, fully guarantee the service of security and user privacy. 


\subsection{Medical Information Platform Construction}

Using advanced cloud computing technology, establish medical cloud, break through the region and time constraints, the medical third-party payment, medical information sharing, referral, medical resource sharing through a unified platform to achieve integration, this is a great breakthrough in the development of medical information. Cloud computing technology is used to build the medical information platform, need to solve the problem is how to integrate the existing types of hospital information system; between the different hospital information sharing and storage problems and how to balance function conflict management mode and management needs.

\subsection{SaaS}

The application of SaaS into medical informatization breaks the taboo that software should be purchased at one time, and thus reduces the construction risks. In this way, the hospital can selectively rent software as per their own practical deman and planning, while the software service supplier, through technical measures, guarantees the security and privacy of medical data, which saves abundant assests that are otherwise used to buy IT products, technology and to maintain operation. The SaaS model is showed in Fig. 2.

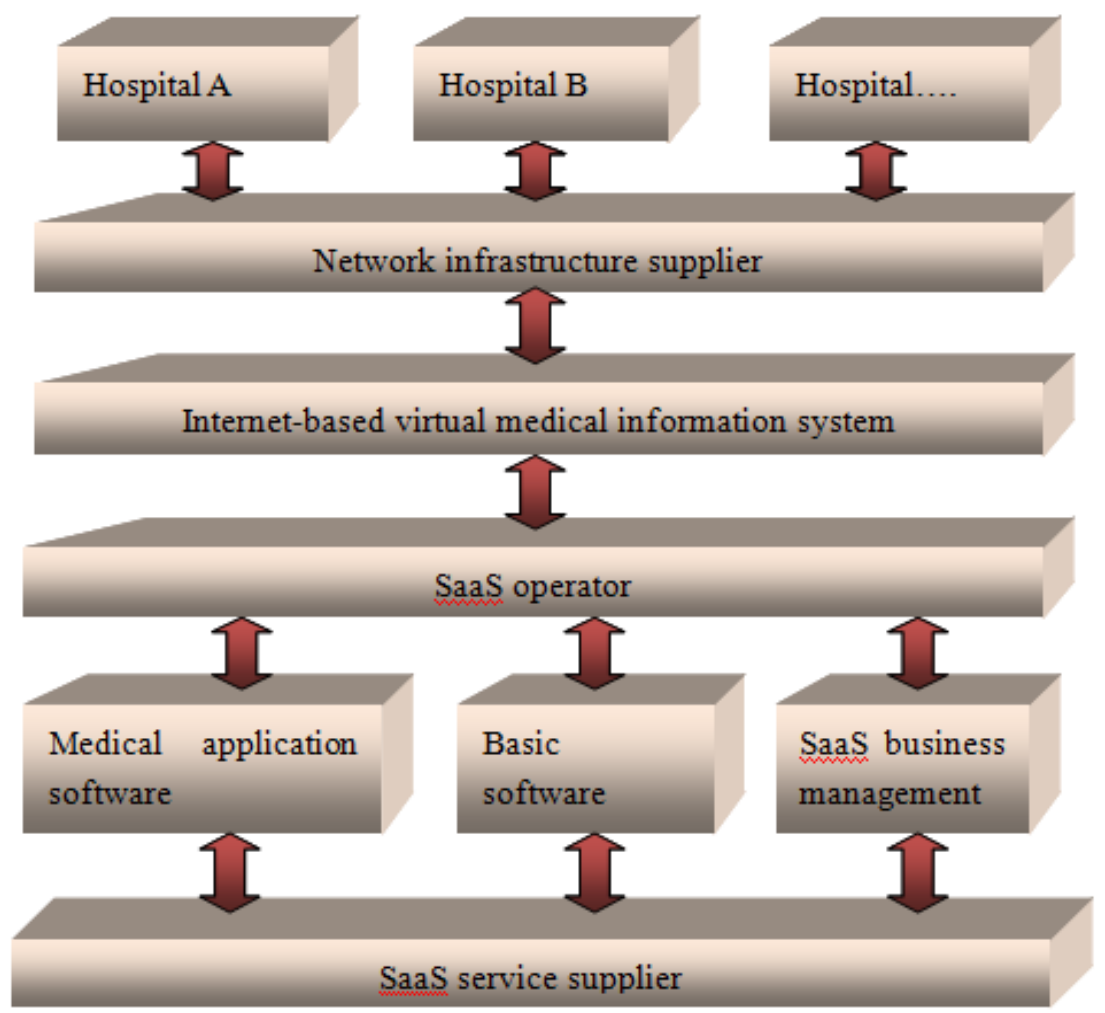

Figure 2. SaaS Model

SaaS, when used into medical informatization, shows some advantages[4]. First, via the innovative mode of SaaS, the software supplier provides a complete set of online services to medical institutions and individuals, including electronic health records (EHRs) and registration appointment. SaaS greatly saves the investment of medical institutions and provides convenience to patients, so that their EHRs can be read and accessed at anytime and anywhere. The software supplier only has to maintain the application software, which saves time and energy and reduces the actual cost of software. Second, the hospital can, depending on the real situation and long-term planning, selectively rent 
relevant informatization software "as demanded", which satisfies the hospital's requirement for information management. In this way, the hospital invests in a relatively low-cost "renting way", alleviating the pressure of insufficient funds, and it reduces the risks of informatization building without consideration of costing depreciation. In this way, hospitals do not have to worry about the tedium, but focus more on improving the working ability and level of medical staff.

\subsection{Space Renting Service from Cloud Storage Services}

In order to guarantee the high availability, high reliability and economy, cloud computing, the distributed storage to store data, the storage redundancy to guarantee the reliability of data storage, is a copy of the same data stored in multiple copies.

Cloud storage means to, through the cluster application, grid technology, or distributed file systems, integrate abundant and diverse storage facilities via application software, so that they provide to the external environment with data storage and business access functions. Data center construction is a main content of digitized hospital building or regional medical information integration. However, independent construction of a safe data center is not easy, but needs abundant prephase investment and professional knowledge, and also subsequent operating costs and maintenance management should never be ignored. Through a high-performance large-capacity cloud storage system, the business operator and IDC data center can provide convenient and rapid space renting seivices to medical units that cannot afford large-capacity storage facilities independently. This satisfies the demand of medical units for the growing data storage and management services. The daily management and maintenance from a large number of professional technicians would guarantee the safe operation of cloud storage systems and prevent the loss of data. The cloud storage services not only provide medical units, community hygiene service stations and individual consumers with storage services, but also contributes to the integrated share of medical information among relevant institutions in a region[5]. The space rent cloud storage service model is showed in Fig. 3.

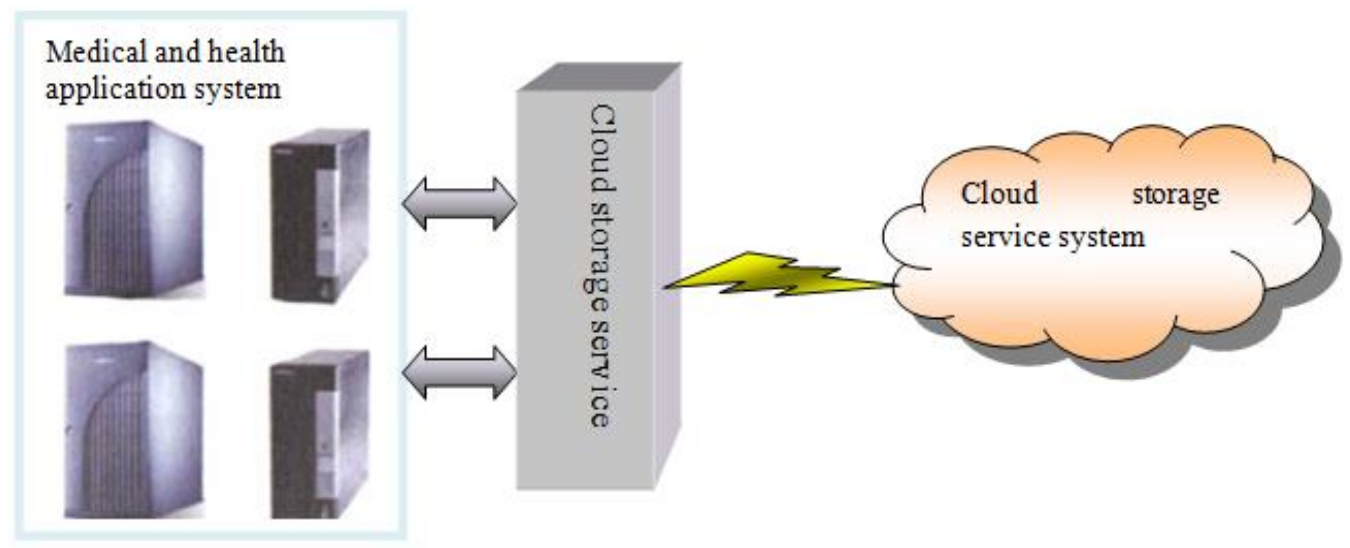

Figure 3. Space Rent Cloud Storage Service Model

\section{Challenges}

Despite many advantages, the cloud computing mode also has some limitations, such as data privacy, use habit, and reliable and stable network transmission.

- Data privacy: How to guarantee the data in the cloud service supplier being private and not illegally used requires both technical advance and further legal improvement.

- Consumer use habits: Altering the consumer use habits, especially many senior medical experts (including senior traditional Chinese doctors and professors), and 
adaptation of consumers to network-based software/hardware application are long-term challenges.

- Reliable and stable network transmission: Cloud computing services depend on networks, so if the network speed is unstable, the performances and stability of diverse services and applications based on cloud computing would be reduced. The popularization of cloud computing relies on the development of network technology.

- Planning for medical cloud capacity: the need to understand or calculate how much of the available capacity of the medical cloud in the data center, the current available capacity, when the release of capacity and other issues. Cloud computing cloud computing allows users to specify the amount of resources needed for their applications to specify the system.

\section{Conclusions and Prospects}

Medical service informatization is an inevitable trend during the new wave of medical reform. By means of informatization, building resource share among medical and health institutions contributes to the optimal integration of medical service resources and the maximum synergy. In the future, the demands for medical software and hardware would be very considerable. Thus, the philosophy and technology of cloud computing that integrate computing resources as services will necessarily alter the developing philosophy and building mode of traditional medical informatization, and plays critical role in the promotion of medical informatization.

In the further reform of the medical system, between the residents and community health service institutions, large hospitals establish a transparent and efficient, inclusive of service platform, so that medical resources further socialization use, more land to promote the further development of the reform of the medical system. The construction of medical information platform will help to solve the problem of information asymmetry in the field of health care in our country, it is helpful to innovate the medical service model, and realize the goal of health insurance.

\section{References}

[1] The NIST Defintion of Cloud Computing(Draft), Special Publication 800-145(Draft), (2011).

[2] E. Bauer and R. Adams, "Reliability and Availability of Cloud Computing", (2014).

[3] B.-Y. Tang, R.-J. Li and J.-H. Lu, "Introduction of Cloud Computing", China, Chemical Industry Press, (2014), pp. 172-176.

[4] X.-P. Hu, Z.-M. Zhang and J.-C. Dong, "Medical Information Research in Cloud Computing Environment", China Digital Medicine, vol. 6, no. 2, (2011).

[5] Z. Ying, Z. Fan and H. Hao, "Application prospect of cloud computing in the development of medical and health information", China Medical Education Technology, vol. 24, no. 4, (2010).

[6] X.-P. Hu, Z.-M. Zhang and J.-C. Dong, "Medical Informatization Based on Cloud Computing concept and technology", Journal of Medical Informatics, vol. 31, no. 3, (2010). 
International Journal of Grid and Distributed Computing Vol. 9, No. 12 (2016) 